\title{
Short Term Outcome and Risk Factors for Mortality in Adults with Criti- cal Severe Acute Respiratory Syndrome (SARS)
}

\author{
HU Xianming (胡先明)' DEVG Yongzhi (爻勇志) ${ }^{2}$, WANG Jun (王 峻) ${ }^{3}$, LI Heping (李和平) ${ }^{3}$, LI Mei (李 梅) ${ }^{3}$, \\ L,U Zuxun (卢祖消) ' \\ 'Department of Social Medicine, School of Public Health. Tongji Medical College, Huazhong University of Science and \\ Technology, Wuhan 430030, China \\ ${ }^{2}$ Department of Cardiovascular Surgery, Union Hospital, Tongji Medical College, Huazhong University of Science and \\ Technology, Wuhan 430030, China \\ ${ }^{3}$ Shanxi Provincial Department of Public Health. Taiyuan 030013, China
}

\begin{abstract}
Summary: The independent risk factors to predict mortality of critical severe acute respiratory syndrome (SARS) were investigated. (nne hundred and two patients diagnosed with critical SARS were admitted to hospitals of Shanxi Province, from March 7, 2003 to June 4, 2003. The patients were prospectively studied after admission to access their short term outcomes and the risk factors associated with adverse outcomes, defined as death. All the demographic and clinical characteristics were studied and univariate and multivariate Logistic regression were employed to access the risk factors. The results showed that of the 102 cases, 23 patients died, with a crude mortality rate of $22.5 \%$. Multivariate Logistic regression revealed that age above 50 [odds ratio (OR) $1.10,95 \%$ confidence internal (CI) 1.03 to $1.16, P=0.004$ ], lymphopenia at early stage (OR $14.62,95 \% \mathrm{CI}$ 1.78 to $11.97, P=0.01$ ) were independently associated with mortality. On the other side, psychotherapy (OR $0.01,95 \% \mathrm{CI} 0.00$ to $0.06, P<0.001$ ) was independently associated with aliveness. It was concluded that critical SARS is a new disease entity that carries significant mortality and morbidity. Specific clinical and laboratory parameters predicting unfavorable and favorable outcomes have been identified.
\end{abstract}

Key words: critical severe acute respiratory syndrome; mortality; independently risk factor; psychotherapy

Severe acute respiratory syndrome (SARS) caused by a novel coronavirus ${ }^{[1}$ 3? has been described in mainland China since March 2003, and several series of patients have been described in the literature ${ }^{[4,5 !}$. Until now, there still no report $\mathrm{re}^{-}$ garding the risk factors associated with mortality of critical SARS and protective effects of psychotherapy. Between March 7, 2003 and June 4, 2003, 102 adults were admitted to SARS control hospitals of Shanxi Province with a diagnosis of critical SARS. A treatment protocol including a combination of antibiotics and systemic steroids was adopted from the beginning of the epidemic, in accordance with the guidelines of the National Department of Public Health of People's Republic of China ${ }^{\left[{ }^{6}\right]}$. Unfortunately, without a definitely reliable treatment regimen (at least in the initial phase), the mortality rate has been high.

We describe the clinical features and short term outcomes of these patients, and attempt to $\mathrm{i}^{-}$ dentify the initial presenting factors that might account for the adverse clinical outcomes.

\section{PATIENTS and METHODS}

\section{1 Patients Selection and Group Classification}

All the patients admitted to the SARS control hospitals of Shanxi province fulfilled the National Department of Public Health criteria for probable

HU Xianming, female, born in 1968, Associate Professor critical SARS were included in the study ${ }^{[7]}$. In brief, the definition of SARS is dyspnea with respiratory rate more 30 times/min, low oxygen saturation with arterial oxygen pressure $\left(\mathrm{PaO}_{2}\right)$ less than $70 \mathrm{mmHg}(1 \mathrm{mmHg}=0.133 \mathrm{kPa})$ or pulse oxygen saturation less than $93 \%$ at $3-5 \mathrm{~L} / \mathrm{min}$ nasal or musk oxygen; or acute lung injury (ALI) or acute respiratory distress syndrome (ARDS) was diagnosed; multi-lobe pulmonary infiltration more than $1 / 3$ or the deteriorated more than $50 \%$ on the chest radiograph in $48 \mathrm{~h}$; shock or multi-organ dysfunction syndrome (MODS); patients with severe comorbidities or combined/complicated with other infection or more than 50-years-old.

All the patients were divided into two groups according to survival or death, i. e. the survival group and the death group. All the demographic and clinical data were prospectively collected and entered into the computer based SARS control database of Shanxi Province. Age, gender, contact history, mean consult time, mean temperature (early stage), important comorbidities (cardiovascular disease, hypertension, diabetes mellitus, chronic obstructive pulmonary disease, asthma, cerebrovascular accident and chronic renal failure, etc), mean fever time (days), recurrent fever, total white cell count at early stage, lymphocyte count at the early stage, pulse oxygen saturation, the time to start steroids after diagnosis, and psychotherapy were analyzed. Both univariate and multivariate Logistic regression were employed to identify the independent risk factors to predict 
death of critical SARS (table 1 ).

Table 1 The demographics and clinical characteristics of all patients

\begin{tabular}{|c|c|c|c|c|}
\hline Variables & Survival group & Death group & $\chi^{2}$ or $t$ values & $P$ \\
\hline \multicolumn{5}{|l|}{ Gender } \\
\hline Male & 35 & 13 & 1.067 & 0.302 \\
\hline Female & 44 & 10) & & \\
\hline \multicolumn{5}{|l|}{ Age } \\
\hline$\leqslant 50 \mathrm{yr}$ & 54 & 8 & 8.423 & 0.004 \\
\hline$>50 \mathrm{yr}$ & 25 & 15 & & \\
\hline \multicolumn{5}{|l|}{ Presence of contact history } \\
\hline Yes & 32 & 15 & 4.378 & 0.036 \\
\hline No & 47 & 8 & & \\
\hline Mean time to consult a doctor (day) & $3 \pm 5$ & $2 \pm 5$ & 1.067 & 0.286 \\
\hline Mean tempcrature $(C \cdot x \pm s)$ & $39.1 \pm 0.58$ & $39.3 \pm 0.57$ & 1.388 & 0.170 \\
\hline \multicolumn{5}{|l|}{ Presence of comorbiditics } \\
\hline Yes & 23 & 12 & 4.203 & 0.04 \\
\hline No & 56 & 11 & & \\
\hline Days of fever & $9.0 \pm 6.0$ & $8.7 \pm 4.0$ & $t=0.204$ & 0.839 \\
\hline \multicolumn{5}{|l|}{ Recurrent fever } \\
\hline Yes & 43 & 10 & 0.856 & 0.355 \\
\hline No & 36 & 13 & & \\
\hline \multicolumn{5}{|l|}{ Total white cell count at carly stage } \\
\hline Decrease & 30 & 4 & 3.614 & 0.164 \\
\hline Normal range & 39 & 16 & & \\
\hline Increase & 10 & 3 & & \\
\hline \multicolumn{5}{|l|}{ Lymphocyte count at early stage } \\
\hline Decrease & 54 & 21 & 4.469 & 0.036 \\
\hline Normal range & 25 & 2 & & \\
\hline \multicolumn{5}{|l|}{ Oxygen saturation } \\
\hline$\leqslant 93 \%$ & 62 & 18 & 27.69 & $<0.001$ \\
\hline$>93 \%$ & 17 & 5 & & \\
\hline \multicolumn{5}{|l|}{ The time to start steroids (day) } \\
\hline$\leqslant 7$ & 61 & 19 & 0.306 & 0.58 \\
\hline$>7$ & 18 & 4 & & \\
\hline \multicolumn{5}{|l|}{ Psychotherapy } \\
\hline Yes & 63 & 1 & 43.323 & $<0.001$ \\
\hline No & 16 & 22 & & \\
\hline
\end{tabular}

\subsection{Data Analysis}

Data were expressed as $\ddot{x} \pm s$ unless specified otherwise. Student $t$ test or Chi-square test was used to compare between groups. Adverse outcome was defined as death. Both univariate and multivariate Logistic regression were employed to identify the independent risk factors to predict death of critical SARS from the variables listed in table 1. SPSS (version 10.0) was used for all statistical analyses.

\section{RESULTS}

\section{1 Demographic and Epidemic Data}

(One hundred and two patients were admitted to the SARS control hospitals between 7th March and 4 th June 2003. Twenty-three patients were died, giving a crude mortality rate of $22.5 \%$. The mean age of the patients was $45.8 \pm 15.4$ years. There were 40 cases $(39.2 \%)$ older more than 50 years and 48 cases $(47.1 \%)$ were males. Thirtyfive cases $(34.3 \%)$ had one or more comorbidities (cardiovascular disease, hypertension, diabetes mellitus, chronic obstructive pulmonary disease, asthma, cerebrovascular accident and chronic renal failure, etc). Forty-seven cases $(46.1 \%)$ had direct contact history. Forty-one cases (40.2\%) consulted to a doctor within 1 to 3 days, 35 cases $(34.3 \%)$ within 4 to 5 days, and 26 cases (25.5 $\%)$ more than 5 days. The mean consult time was 
3. $6 \pm 3.2$ days. Once a patient consulted to a doctor and diagnosed as SARS, he or she was immediately admitted to the SARS control hospital, so the consult time is the admission time. The age, contact history, important comorbidities were significant difference between groups $(P<0.05)$; while in gender and mean consult time no significant difference was found between groups $(P>0.05$, table 1).

\section{2 Clinical Characteristics}

The presenting symptoms included fever 102 cases $(100 \%)$, chills 21 cases $(20.6 \%)$, cough 54 cases $(52.9 \%)$, dyspnea 43 cases $(42.2 \%)$, diarrhea 8 cases $(7.8 \%)$, myalgia 4 cases $(3.9$ $\%)$. Thirty cases $(29.4 \%)$ had the temperature between 38.0 to $38.9{ }^{\circ} \mathrm{C}, 63$ cases $(61.8 \%)$ be- $^{-}$ tween 39.0 to $39.9{ }^{\circ} \mathrm{C}$, and 9 cases $(8.8 \%)$ more than $40{ }^{\circ} \mathrm{C}$; Mean fever time lasted for $9.0 \pm 5.6$ days. Fifty-three cases $(52.0 \%)$ had recurrent fever. There were no significant difference regarding temperature, mean fever time, and recurrent fever between groups $(P>0.05$, table 1$)$.

\subsection{Laboratory Test}

Common presenting laboratory findings included total white cell count decreased in 34 cases $(33.3 \%)$, in normal range in 55 cases $(53.9 \%)$, and increased in 13 cases $(12.7 \%)$; the lymphocyte count decreased in 75 cases $(73.5 \%)$; the oxygen saturation less than $93 \%$ in 80 cases ( 78.4 $\%)$. There was no significant difference in total white cell count $(P>0.05)$, but lymphocyte count $(P=0.04)$ and oxygen saturation $(P<0.001)$ had significant difference between the survival and death groups (table 1).

\section{4 Steroids and Psychotherapy}

More than $90 \%$ of all cases received steroids therapy. In 80 cases $(78.4 \%)$, no steroids was used or the use of steroids time lasted less than 7 days, while 22 cases $(21.6 \%)$ used steroids more than 7 days. Sixty-four cases $(62.8 \%)$ received psychotherapy. There was no statistical difference in the time to start steroids $(P=0.58)$, while there was significant difference in the psychotherapy between the two groups $(P<0.001$, table 1$)$.

\subsection{Short Term Outcomes}

Seventy-nine cases $(77.5 \%)$ were discharged and 23 cases were died, giving a crude mortality rate of $22.5 \%$.

\section{6 Factors Affecting Mortality Rate}

The univariate Logistic regression showed that the mortality risk was greatly increased in those aged above 50 , presence with important comorbidities, direction contact history, lymphopenia at early stage, and lack of psychotherapy (table 2).

The multivariate non-conditional Logistic regression model was used to look for independent risk factors for mortality. Using a model with age above 50 , presence of important comorbidities, direction contact history, lymphopenia at early stage, and lack of psychotherapy, it was found that the age over 50 (OR k. $10,95 \%$ CI 1.031. 16, $P=0.004)$ and the presence of lymphopenia at early stage (OR $14.62,95 \% \mathrm{CI} 1.78-11.97, P$ $=0.01$ ) were all independent predictors of death; On the other side, psychotherapy (OR $0.01,95 \%$ CI 0.00 to $0.06, P<0.001)$ was independently associated with aliveness (table 3 ).

Table 2 Analysis of factors affecting mortality using the univariate Logistic Regression

\begin{tabular}{lcccccc}
\hline \multicolumn{1}{c}{ Variables } & $B$ & Standard error & $\chi^{2}$ & $P$ & OR & $95 \% \mathrm{CI}$ \\
\hline Age $>50 \mathrm{yr}$ & 0.06 & 0.02 & 10.04 & 0.002 & 1.06 & $1.03-1.16$ \\
Presence of contact history & 1.01 & 0.49 & 4.20 & 0.04 & 2.75 & $1.0-4.67$ \\
Presence of comorbidities & 0.98 & 0.49 & 4.05 & 0.04 & 2.66 & $1.0-8.0$ \\
Lymphopenia at admission & 1.53 & 0.78 & 3.86 & 0.05 & 4.63 & $0.0-0.06$ \\
Presence of psychotherapy & -4.46 & 1.06 & 17.71 & $<0.001$ & 0.01 & $1.78-11.97$ \\
\hline
\end{tabular}

Table 3 Multivariate analysis showing the independent risk factors for mortality using Logistic Regression

\begin{tabular}{lrrrrrr}
\hline \multicolumn{1}{c}{ Variables } & B & Standard error & $\chi^{2}$ & $P$ & OR & $95 \% \mathrm{CI}$ \\
\hline Age $>50 \mathrm{yr}$ & 0.09 & 0.03 & 8.08 & 0.004 & 1.10 & $1.03-1.16$ \\
Lymphopenia at admission & 2.68 & 1.07 & 8.25 & 0.01 & 14.62 & $1.78-11.97$ \\
Presence of psychotherapy & -5.19 & 1.24 & 17.52 & $<0.001$ & 0.01 & $0-0.06$ \\
\hline
\end{tabular}

\section{DISCUSSION}

This study aimed to determine the short term outcomes and risk factors on presentation that were associated with an adverse clinical course and mortality in a group of Chinese patients with critical SARS. Age above 50 was associated with a higher rate of mortality. The results were consistent with other reports ${ }^{[8,9]}$. Some reasons may explain why the older patients tender to become critical cases and predispose to death: firstly, multi-or- 
gans function of aging patients is decreased, with limited cardiopulmonary compliance and reserve, less adopt ability to intcrnal and external environment, easy to occur respiratory failure, cardiac failure and imbalance of electrolytes; secondly, the immune response of the aging patients is decreased with less immature lymphocytes, less ability of macrophage to get rid of pathogens, and the decreased secretive ability of lymphocytes to produce special antibody with the increase of age; thirdly, aging patients usually suffer from comorbidities. In this study, 35 cases $(34.3 \%)$ suffered from important comorbidities, and the latter is an independent risk factor of SARS with the end point being death ${ }^{[8]}$. Finally, aging patients tender to bc delayed diagnosis and treatment due to atypical symptoms. Attention should be paid to aging critical SARS patients, especially to those patients having comorbidities. I.ymphopenia in the carly stage, including the reduction of absolute lymphocyte count and the relative percentage of lymphocyte of the whole white cell, especially the reduction of $\mathrm{CD}^{+}, \mathrm{CD} 88^{\circ}$, and $\mathrm{CD} 3^{\prime}$ lymphocytes, in dicates coronavirus may destroy lymphocytes in an acute process. Study by other authors had shown that CD3' cells were significantly reduced in the early stage, reached the bottom during the critical stage and lasted to death" The results of this study demonstrated that lymphopenia was inde pendently associated with mortality. For this reason, in the treatment of critical $S \wedge R S$ patients, steroids should be under control with indications, and prophylactic use of antibiotics may contribute to prevention of secondary infections.

Over psychological response causes over extension of sympathetic vasoconstrictor nerve and the increase of catecholamine, as a result, the whole system vascular bed constricts and amount of blood transfers to pulmonary circulation, the acute increase of pulmonary volume further reduces the lung compliance, increases the dyspnea, and increases the difficulty to control pulmonary infection. Because of the characteristics of SARS such as high infection, congregation. and the rapid progress to death of partial patients. many patients with SARS have severe phobia, such as insomnia, poor appetite, palpitation, and repeatedly consult to medical officer, etc. For these reasons, medical staffs should carefully explain the clinical and epidemical characteristics, as the optimistic emotion can increase the immune ability, thus to improve clinical outcomes. The current study demonstrates that psychotherapy in the early stage is associated with reduced mortality of critical SARS.

\section{REFERENCES}

1 Ksiazek T ( $r$, Erdman D. Goldsmith C S et al. A novel coronavirus associated with severe acute respiratory syndrome. V Eng! I Med, 2003.348:1953

2 Drosten C, Gunther S, Prciser W et al. Identification of a novel virus in patients with severe acute respiratory syndrome. N Engl J Med, 2003,348:1967

3 Peiris J S M. Lai S T. Poon I, I, M et al. Coronavirus as a possible cause of severe acute respiratory syndrome. Lancet, 2003,361:1319

4 Zhao Z I., Zhang F C. Xu M et al. Clinical analysis of 190 cases of outbreak with atypical pneumonia in Guangzhou in spring. Chin Med J (Chinese), 2003,83:713

5 liu Z Y, Li T S. Wang $Z$ et al. Clinical features and therapy of 106 cases of severe acute respiratory syndrome. Chin J Inter Med (Chinese). 2003.42:373

6 National Disease Control Centcr of China. National Department of Public Health of People's Republic of China. The clinical diagnosis critcria for severe acute respiratory syndrome (Chinese). http://www. laben. $\mathrm{com} / \mathrm{show}$ aspxid $=2718$. $\mathrm{cid}=22$.

7 Vational Disease Control Center of China. National Department of Public Health of People's Republic of China. The recommended treatment protocol and discharge criteria for severe acute respiratory syndrome or probable severe acute respiratory syndrome. http://news. sina. coml. cn/ci2003-04-06/1646983385. shtml.

8 Chan J W M, Ng C K, Chan Y H et al. Short term outcome and risk factors for adverse clinical outcomes in adults with severe acule respiratory syndrome (SARS). Thorax, 2003.58:686

9 Jiang $\mathrm{T} J$, Zhou X Z. Zhao $M$ et al. Analysis of severe acute respiratory syndrome in Beijing. Chin J Inter Med (Chinese) 2003.42 .369

10 Zhao C H, Guo Y B, Wu H et al. Clinical manifestation, treatment, and outcome of severe acute respiratory syndrome: analysis of 108 cases in Beijing. Chin Med J (Chinese) , 2003,83:897

(Received Sep, 29,2003) 